\title{
“OBSERVATORIO DE TURISMO". UN ESPACIO DE ANÁLISIS Y COMUNICACIÓN COMO CONTRIBUCIÓN PARA EL DESARROLLO DEL TURISMO EN EL ECUADOR
}

\author{
Patricia Carrera Burneo \\ Pontificia Universidad Católica del Ecuador \\ pcarrera@puce.edu.ec \\ Santiago López \\ Pontificia Universidad Católica del Ecuador \\ nslopez@puce.edu.ec
}

\section{RESUMEN}

A nivel mundial, a pesar de las múltiples crisis internacionales, durante los primeros nueve meses del 2013 el turismo creció a nivel mundial en un 5 por ciento rebasando las expectativas del sector (OMT, 2013). El Ministerio de Turismo del Ecuador (2013) reportó que esta actividad económica representó en el 2012 el 5 por ciento del PIB ecuatoriano, por lo que el Gobierno del Ecuador ha decidido que uno de los sectores productivos a desarrollarse será el turismo, declaración inédita y coyuntural que requiere ser tomada en cuenta por la academia.

El conocimiento es el factor clave para el desarrollo y la sustentabilidad de cualquier sector económico y social, la creación de un observatorio para la divulgación de información generada en espacios académicos y especializada en turismo es necesaria porque Ecuador adolece de información básica y especializada que puede contribuir al mejoramiento de la educación, producción y competitividad del sector turístico ecuatoriano.

La instauración de un Observatorio en turismo para investigar e informar sobre la realidad académica, social y económica relacionada con el sector turístico del Ecuador, requiere de una base conceptual y metodológica que fundamente el diseño del observatorio de turismo, su estructura y funciones deben además obedecer a la realidad académica, social y económica ecuatoriana.

Palabras claves: observatorio de turismo, conocimiento, información, desarrollo sustentable, sector turístico, diseño. 


\title{
“TOURISM OBSERVATORY" A TOOL FOR TOURISM ANALYSIS AND DEVELOPMENT IN ECUADOR
}

\author{
Patricia Carrera Burneo \\ Pontificia Universidad Católica del Ecuador \\ pcarrera@puce.edu.ec \\ Santiago López \\ Pontificia Universidad Católica del Ecuador \\ nslopez@puce.edu.ec
}

\begin{abstract}
In spite of multiple international crises, during the first nine months of 2013, the tourism industry grew by 5 percent worldwide and overcoming the expectative for the sector (OMT, 2013). The Ministry of Tourism of Ecuador reported that tourism represented 5 percent of the Ecuadorian GDP in 2012 and announced that the government had decided to promote the development of the tourism sector in the country. This relevant announcement does not have precedent and needs to be taken into account by academia.
\end{abstract}

Knowledge is a key factor to promote development and sustainability of any economic and social sector. In this context, this article aims to propose the creation of an Observatory in tourism in order to monitor development in the country. Observatories are key motors for spreading information generated by academia and other actors in tourism. This is a key aspect considering that Ecuador lacks basic and specialized information that could contribute to the improvement of education, productivity and competitiveness of the Ecuadorian tourism sector.

The establishment of a tourism observatory for conducting research and providing information regarding tourism in Ecuador, requires clear and solid conceptual and methodological basis. In this context, the paper aims to provide some considerations regarding the creation of an observatory, its structure and functions based on the country's academic, social and economic context.

Key words: tourism observatory, knowledge, information, sustainable development tourism sector, design. 


\section{INTRODUCCIÓN}

El avance vertiginoso de la ciencia y la tecnología en el siglo 20 y 21 son atributos para que al mundo en la actualidad se le atribuya el nombre de sociedad del conocimiento, uno de los factores más importantes para el desarrollo de ésta sociedad se debe al uso que se le atribuya al conocimiento.

Un mundo globalizado por la comunicación tecnológica no tiene límites en la difusión de la información, el uso efectivo de ésta crea riqueza y poder en las naciones, el conocimiento y la información pasaron a integrar parte del capital económico y financiero y por tanto están inmersas dentro de la lógica del mercado. La utilización y reproducción del conocimiento por los individuos está limitado por quienes lo producen.

Las nuevas tecnologías han facilitado el acceso a la información, este proceso se opera sin espacio ni tiempo, no existen fronteras ni pasado ni futuro, solo existe el aquí y el ahora (Tünnermann y de Souza Chaui, 2003).

La explosión del conocimiento es otra característica del siglo 21, el conocimiento producido es complejo y tiene una rápida obsolescencia, esto desencadena incertidumbre y como respuesta la integración de varias disciplinas para la generación del conocimiento (Escotet, 2002).

Gibbons (1997) expresa que no solo surgen nuevas representaciones de conocimiento sino que están cambiando también las formas de producción y diseminación. La forma contemporánea de generar conocimiento se caracteriza por la intervención de múltiples actores lo que se traduce en una responsabilidad compartida, en un proceso socialmente distribuido.

Se reconoce a la Universidad como una institución generadora de conocimientos, por lo que ésta se enfrenta cada día a cambios repentinos y vertiginosos que demandan que reaccione ante una diversificación de las sociedades, masificación de la educación superior, incorporación de nuevas tecnologías de comunicación, nuevas demandas de trabajo que requieren que los profesionales tengan iniciativa, corresponsabilidad en las decisiones, interdisciplinaridad en los puestos de trabajo, movilidad de los perfiles profesionales (Escotet, 2002).

La sociedad contemporánea demanda respuestas, por ello la Universidad debe involucrarse en los procesos sociales, económicos y culturales; es inminente su transformación para participar en el destino de un pueblo, debe encontrar mecanismos o instrumentos para comunicarse con la sociedad. 
Un mecanismo para acercarse a la realidad social, económica y cultural de un pueblo, a través de investigaciones interdisciplinarias, en red, para contribuir con la identificación de problemas específicos de la educación superior, la generación y uso de la información son los observatorios, que se definen como espacios de encuentro y dialogo, fundamentándose en investigaciones de temas o problemas de la realidad social con diversidad de métodos, técnicas de investigación y análisis de datos (Phélan, 2007).

La propuesta de constituir un "Observatorio de Turismo" nace de la necesidad de reflexionar y analizar sobre la mejora de una actividad económica como el turismo que es importante a escala mundial, regional y local y que se sostiene en el talento humano que planifica, gestiona y opera este sector económico.

El "Observatorio de Turismo" se origina en el seno de la Escuela de Hotelería y Turismo (EHT) de la Pontificia Universidad Católica del Ecuador (PUCE) con el objetivo de proporcionar indicadores de gestión y herramientas metodológicas para mejorar la educación que imparte la EHT, basándose en el monitoreo, seguimiento y evaluación de indicadores de calidad proporcionados por el Consejo de Evaluación, Acreditación y Aseguramiento de la Calidad de la Educación Superior (CEAACES). Con el objetivo de vincularnos con la colectividad, con el sector público y privado del turismo ecuatoriano, se generará información sobre indicadores relacionados con la productividad y competitividad del sector turístico y, por último, otro eje importante es investigar sobre la articulación de las políticas públicas entre el sector público y privado. En este esfuerzo se pretende trabajar con otras universidades del país.

\section{Antecedentes}

Desrosières (1996) del Centro de Investigación en Economía y Estadística (INSEE) de París señala que los observatorios económicos regionales nacen en la década de los sesenta, creados por el INSEE y por la Delegación de Ordenamiento del Territorio y Acción Regional (DATAR) de Francia. El concepto de "observatorio" como se lo conoce en la actualidad se desarrollan en la década de los noventa por los gobiernos y comunidades locales con el objetivo de construir indicadores, analizarlos y debatirlos entre los diferentes actores de sectores importantes de una sociedad como son la salud, educación, ambiente, empleo, etc.

Los observatorios, en la actualidad, son escenarios de debate social, científico, ambiental, económico, sustentados en diversos tipos de información generada por la investigación científica, social y económica financiera. La información generada es utilizada para la toma de decisiones de los diferentes actores sociales, organizándose alrededor de éste en redes tanto de investigadores, que proveen información, como de usuarios que la manejan y gestionan. 
A nivel mundial existen un sinnúmero de observatorios que tienen funciones que van desde la simple recolección y divulgación de datos estadísticos hasta la intermediación e intervención directa en problemas sociales.

En Latinoamérica, la Clacso (2013) dispone de un Portal de Observatorios en el cual constan 156 a nivel mundial que tienen como objetivo observar la realidad social, económica y ambiental de diferentes naciones.

En cuanto al turismo como actividad económica, a nivel mundial la mayoría de los países y sus capitales tienen observatorios turísticos que básicamente contienen estadísticas y algunas investigaciones relacionadas con el turismo.

El Ecuador registra un número variado de observatorios sobre todo en temas sociales: en Democracia y Control Institucional: Observatorio de la Política Fiscal; en Conflictividad Social y Medio ambiente: Acción Ecológica; en Comunicación: Fundación Andina para la Observación y Estudios de Medios de Fundamedios; en Educación Infancia y Juventud el Observatorio de los Derechos de la niñez y la adolescencia; de Movilidad Académica el de la Universidad Técnica de Loja (UTPL); en Medio Ambiente: de Política Ambiental. La Universidad Andina Simón Bolívar, Sede Ecuador, creo en el 2010 un Observatorio de la Pequeña y Mediana Empresa, cuyo principal objetivo es difundir información sobre la realidad de la PyME.

En cuanto a observatorios de turismo en el Ecuador solo se registró en la página institucional del Ministerio de Turismo del Ecuador (MINTUR) un Observatorio de Galápagos en el cual solo existe un documento de estadísticas sobre el turismo en las islas.

Por lo expuesto, en el Ecuador no existen observatorios relacionados con el turismo y resulta imprescindible contar con este constructo social que bien puede nacer en la academia para contribuir con el desarrollo del turismo como actividad económica importante, ya que así lo ha declarado el Gobierno Ecuatoriano al incluirlo en los 14 sectores productivos a desarrollar. Por lo expuesto, es necesario observar el turismo desde diferentes aristas que no solo contribuyan con el análisis del sector productivo sino con el educativo generador del talento humano en el cual se soporta cualquier actividad económica y social. 


\section{Justificación}

A nivel mundial, a pesar de las múltiples crisis internacionales, en el 2012 las llegadas de turistas internacionales crecieron en un 4\% superando los 1000 millones de turistas. La región de Asia y el Pacífico incremento las llegadas en un 7\%, fue la región de mayor crecimiento, seguida por África con un 6\%, las Américas reportaron un 5\% y, el Oriente Medio un 5\%. América del Sur registró un crecimiento del 5,3\%, Ecuador reportó en el 2010: 1'.047 mil turistas internacionales, durante el 2011: 1'141 mil turistas internacionales y en el 2012: 1'272 mil turistas (OMT, 2012). ecuatoriano.

El MINTUR (2013) reporta que el turismo representó en el 2012 el 5\% del PIB

De acuerdo con el Foro Económico Mundial (WEF, 2013) en el informe correspondiente al Índice de Competitividad de viajes y turismo, Ecuador ocupa en el 2012 el puesto 14 entre 27 países americanos después de Chile, Argentina y Perú. Indicadores relacionados con la infraestructura y recursos humanos tienen una puntuación baja, sin embargo durante el 2012 el turismo contribuyó con 282.000 empleos, cifra que representa el 4,6\% del total de empleos generados en el Ecuador y tiene una proyección de crecimiento del $3 \%$ anual.

El Gobierno Ecuatoriano a través de la Secretaria de Planificación y Desarrollo (Senplades, 2013) plantea transformar la matriz productiva del país a través de la diversificación de la producción, basada en el desarrollo de 14 sectores productivos, en los que se encuentra el turismo y 5 industrias estratégicas.

El Gobierno actual reconoce que nuestra economía se basa en la exportación de bienes primarios que carecen de valor agregado como son la tecnología u otras expresiones del conocimiento; persiste hasta la presente fecha un modelo primario exportador con el consecuente riesgo que a este tipo de economías le caracteriza: variación de precios de materias primas internacional, desbalance entre importaciones y exportaciones, desempleo, entre otros indicadores. El Senplades (2013) propone cuatro ejes para la transformación de la matriz productiva cuya base es la generación de conocimiento y tecnología para las industrias y sectores estratégicos propuestos.

Es indudable que el conocimiento es el factor clave para el desarrollo y la sustentabilidad de cualquier sector económico y social, la creación de un observatorio para la divulgación de información generada en espacios académicos y especializada en turismo se justifica porque Ecuador adolece de información básica y especializada que bien utilizada puede contribuir al mejoramiento de la producción y competitividad del sector turístico ecuatoriano. 


\section{Objetivo general}

Proponer la creación e implementación de un Observatorio en turismo para investigar e informar sobre la realidad académica, social y económica relacionada con el sector turístico del Ecuador, contribuyendo para el desarrollo del país.

\section{MARCO CONCEPTUAL}

\section{Concepto de observatorio}

Los observatorios son instrumentos que recogen, producen y analizan datos relacionados con áreas como la economía, sociedad y territorio, con el objetivo de conocer y dar luces sobre problemas actuales, recurren a metodologías específicas que giran en torno al tratamiento de datos y a la integración de la información, utilizan metodologías cuantitativas y cualitativas para el análisis e interpretación de datos y construyen indicadores específicos. En términos organizativos, asignan importancia a su relación con los organismos productores de datos, tanto de carácter público como privado (Phélan, 2007).

"Los observatorios, no son instancias vigilantes, tampoco fiscalizadoras, sino que se conciben como estructuras especializadas de apoyo, cuya rigurosidad técnicoacadémica de trabajo apoye la transparencia y neutralidad de las acciones, garantizando objetividad en la información y el conocimiento que se emane de estos" (Malagón, 2010).

Para Muñoz y Torrent (2003) un observatorio es un instrumento que genera y gestiona información fundamental para la planificación y colabora con la colectividad para que esta se constituya una comunidad de aprendizaje e innovación.

Según Farnés (2011) "es un conjunto dinámico, porque la expresión de sus actividades y productos cambia constantemente dependiendo de las circunstancias y de los intereses del observatorio, se adapta a los nuevos requerimientos y sufre mejoramientos y revisiones incesantes. Fundamentalmente, el origen de esta dinámica radica en la relación información/monitoreo/investigación".

A manera de corolario, se puede decir que un observatorio es una iniciativa permanente, de carácter multidisciplinario, que investiga, monitorea y evalúa diferentes temáticas dentro del ámbito social, económico, ambiental o político, con esta información es capaz de generar un sistema de información cuyos datos se comunican a través de diferentes medios para contribuir con el desarrollo de una comunidad local, nacional, regional o mundial. 


\section{Objetivos y funciones de los observatorios}

Un observatorio puede iniciar su organización y estructura con acuerdos de voluntades entre instituciones que se las denomina fundadoras. Muchos de los observatorios tienen personería jurídica y un patrimonio propio con autonomía administrativa y financiera para apoyar las funciones que se les ha asignado.

De acuerdo con la bibliografía consultada los propósitos de los observatorios son innumerables y naturalmente dependen del tema a observar, sin embargo existen objetivos generales que son comunes y que caracterizan a los observatorios:

- Proporcionar a la sociedad información relacionada con un tema de interés local, nacional, regional para propiciar aprendizaje, innovación y actividades de gestión, entre otras, en la sociedad.

- Contribuir con el desarrollo de metodologías de investigación que permitan la agregación de información a lo largo del tiempo, con el fin de analizar en forma secuencial y sistemática los datos para que los usuarios puedan proyectar y planificar acciones y en última instancia tomar decisiones.

- Convocar a otros actores del sector (económico, social, ambiental) para que contribuyan con información valiosa para la comunidad (datos, experiencias, investigaciones).

- Propiciar espacios de reflexión con el propósito de alcanzar igualdad de oportunidades.

Por tanto, las funciones que todo observatorio debe cumplir son:

- Generación, monitoreo y evaluación de información relevante para la comunidad que tiene interés en las diferentes temáticas. Construcción de un sistema de información.

- Desarrollo de metodologías de investigación.

- Divulgación de la información a través de diferentes medios de comunicación.

- Ser en un mecanismo de dialogo entre los distintos actores del sector que se observa.

\section{Estructura básica de un observatorio}

De acuerdo con la bibliografía analizada, la mayoría de los observatorios tienen una estructura de carácter funcional simple, sus componentes forman un sistema interconectado, es horizontal y sus partes cumplen las funciones básicas de un observatorio que se circunscriben a investigar, monitorear, evaluar y comunicar información pertinente. 
El Observatorio Económico y Social de Terrassa-Cataluña (Muñoz y Torrent, 2003) señala una estructura básica (Fig, No.1) que todo observatorio debe poseer para cumplir con sus funciones, a continuación se describen cada uno de los componentes:

Figura $\mathrm{N}^{\circ} 1$. Estructura básica de un observatorio

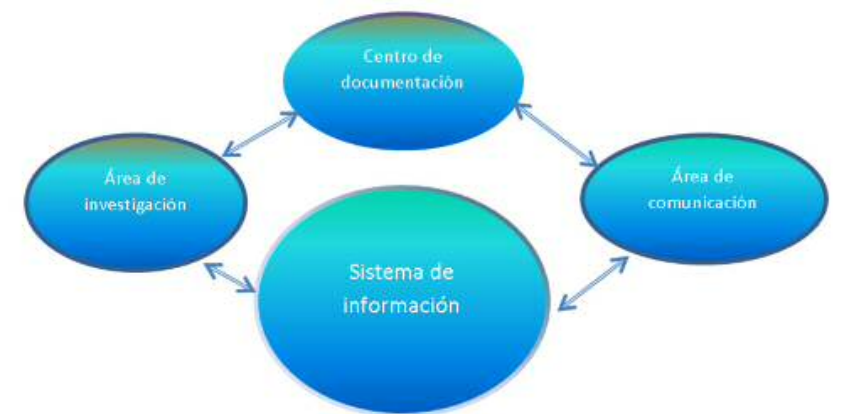

Fuente: Observatorios socioeconómicos locales y planificación estratégica (Muñoz y Torrent, 2003).

Sistema de información: contiene la información: datos, indicadores sociales, económicos, ambientales etc., que se han generado y validado por profesionales que constituyen el Área de Investigación y finalmente serán comunicados a la comunidad por profesionales del Área de comunicación.

Área de investigación: conformado por profesionales altamente capacitados que investigan sobre la problemática que se observa y que obedecen a los objetivos de un observatorio.

Área de comunicación: formada por profesionales capacitados en la difusión de información: expertos en tecnologías de la información y/o comunicación, relaciones públicas, diseño gráfico, etc., que tienen como función comunicar los resultados de las investigaciones a la sociedad.

Centro de documentación: espacio virtual o físico que recepta y almacena la información primaria y secundaria generada por el Área de Investigación y divulgada por el personal del Área de comunicación a través del Sistema de información. 


\section{PROPUESTA PARA EL DISEÑO DE UN OBSERVATORIO}

\section{Diseño del Observatorio en Turismo}

El observatorio de turismo tiene como objetivo generar investigación aplicada en diferentes temas relacionados con el sector turístico (educación superior, productividad, competitividad, políticas públicas) por lo que se lo ha diseñado como una herramienta de generación de información para que los diferentes actores del sector la utilicen en función de sus necesidades.

Figura N 2: Etapas metodológicas para la implementación del Observatorio en Turismo.

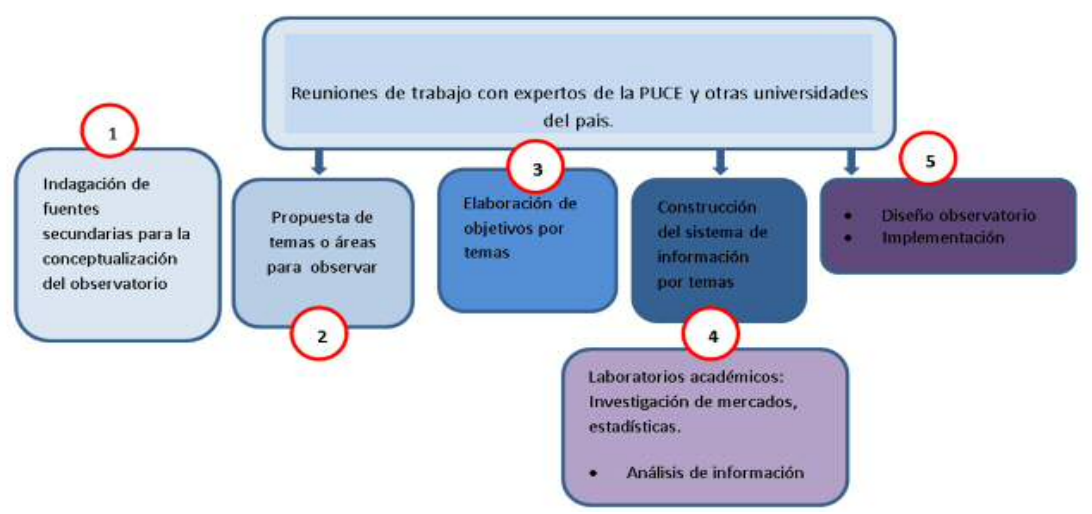

Fuente: Observatorio de la Universidad de Costa Rica (citado por Malagón, 2010).

Para diseñar el Observatorio de Turismo de la EHT de la PUCE se tomó como base la metodología para la implementación de observatorios de la Universidad de Costa Rica (citado en Malagón, 2010) la que fue adaptada a los requerimientos de la EHT de la PUCE, se impuso un proceso lógico y sistemático que se describe a continuación:

1. Se inicia con la indagación de fuentes secundarias para tener una idea clara sobre la problemática a investigar (conceptualización de observatorios). Se analiza la problemática en reuniones de trabajo con expertos.

2. Propuesta de temas de investigación relacionados con el sector turístico: Se reconoce que un observatorio es un espacio que investiga, monitorea y evalúa indicadores que sirven para la planificación y gestión de actividades, por lo que se propone temas a indagar que están relacionados con la ausencia de 
información sobre turismo en Ecuador y con las fortalezas de las unidades académicas de cada universidad participante.

3. Formulación de los objetivos del observatorio: las reuniones con expertos en turismo y en otras áreas del conocimiento son la base para la formulación de los objetivos.

4. Propuesta y discusión de un sistema de información cuyos datos son obtenidos de las investigaciones de los profesores de la PUCE y de otras universidades participantes, así como de los laboratorios académicos dirigidos por profesores de las distintas materias que componen la malla curricular de una carrera. Una vez definidos los temas específicos a investigar, tanto profesores como estudiantes de las asignaturas relacionadas con la Estadística e Investigación de Mercados de las carreras de turismo y hotelería aplicarán herramientas para la recolección y análisis de datos. Los informes serán preparados por los profesores y entregados al área de investigación del Observatorio para su validación y difusión.

5. Paralelamente al paso No.4 se propone el diseño operativo del observatorio, los expertos acogen la propuesta y se lo implementa para la difusión de la información generada por los laboratorios y validada por expertos.

\section{Estructura y funciones del Observatorio de Turismo de la EHT de la PUCE}

Para Fazenda (2013) el siglo 21 representa una oportunidad y un desafío para los observatorios de turismo por varias razones, entre las más importantes señala: crisis económicas internacionales, desarrollo de economías de países emergentes (China, India, Rusia, Brasil), alteraciones en los mercados y en los perfiles de los turistas, creciente importancia en la sostenibilidad del turismo, alteraciones climáticas y desastres naturales envejecimiento de la población, crecimiento del turismo senior.

Ante un escenario incierto, Fazenda (2013) expresa que los observatorios deben proporcionar conocimiento para una mayor competitividad de destinos y empresas, posibilitar información para mejorar la calidad de la oferta, apoyar la toma de decisiones basándose en el análisis de los indicadores claves del sector turístico.

La globalización, los cambios repentinos en la economía y estructura social de los países requiere de un proceso investigativo sistémico y sostenido en las diferentes áreas del turismo, pero que además los resultados de las investigaciones aplicadas sean de fácil acceso y actualizados en forma permanentemente, características que deben tenerse en cuenta al momento de crear un observatorio. 


\section{Objetivos del Observatorio}

El Observatorio de Turismo de la EHT de la PUCE tiene los siguientes objetivos:

Objetivo General: Generar un sistema de información para el sector turístico que contribuya al mejoramiento de la educación, de la productividad y competitividad del sector turístico.

\section{Objetivos Específicos:}

- Contribuir con investigaciones y prácticas académicas para el mejoramiento de la calidad de la Educación Superior en turismo.

- Contribuir con el mejoramiento de la productividad y competitividad del sector turístico ecuatoriano a través de la indagación de indicadores que revelen la estructura y dinámica del mercado turístico interno.

- Investigar la articulación que existe entre las políticas públicas relacionadas con el turismo y la realidad de este sector.

\section{Estrategias para consolidar los objetivos:}

- Gestión para que otras universidades con escuelas o facultades de turismo y hotelería a nivel nacional participen en la creación de un observatorio.

- Gestión interna, en las unidades académicas de hotelería y turismo de las universidades participantes promover la consolidación de laboratorios académicos para que a través de la indagación, compilación y análisis de datos relacionados con la productividad y competitividad del sector turístico se cumplan con los resultados de aprendizaje planteados en cada una de las asignaturas que intervienen.

- Comunicar resultados a las diferentes instancias públicas y privadas con el objetivo de que se unan en el esfuerzo.

\section{Estructura y funciones del Observatorio en Turismo}

La estructura propuesta (Fig. No.3) tiene en primer lugar una instancia que se encarga de la Dirección del Observatorio, cumple funciones de gestión, organización y coordinación de actividades tanto con el sector interno de la EHT como con el externo, es decir, con otras universidades del país y entidades del sector público y privado.

Inmersa en la estructura interna del observatorio se encuentran las áreas de investigación y comunicación para conformar el sistema de información. 
Figura $\mathrm{N}^{\circ} 3$ Estructura del Observatorio en Turismo de la EHT de la PUCE
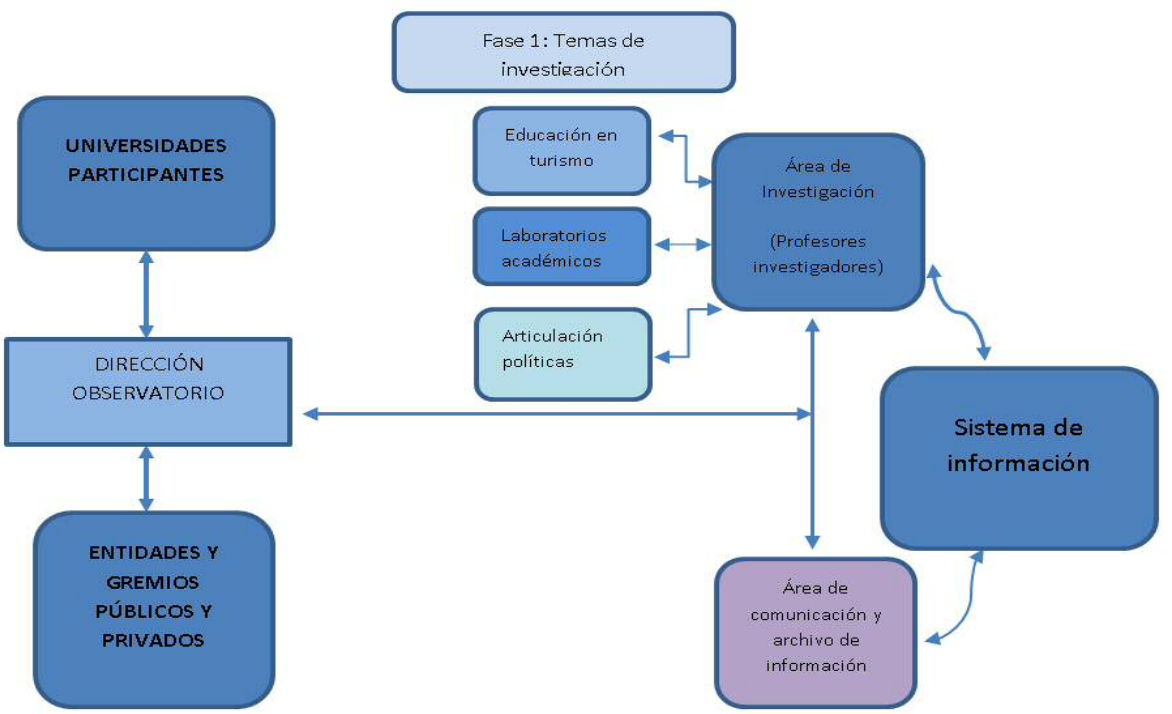

Fuente: elaboración propia

El área de investigación está conformada por profesores que serán responsables de los temas de investigación propuestos en la Fase I del observatorio. Un profesor coordinará la generación, compilación y análisis de información con los laboratorios académicos, espacios que generan información relativa a las materias que conforman el pensum de las carreras de turismo y hotelería de la EHT y de otras universidades del país.

El área de comunicación estará a cargo de un profesor experto en tecnologías de la información, al inicio se trabajará con un aula virtual, en esta se colocarán los resultados de las investigaciones de los laboratorios e información pertinente con los objetivos del observatorio durante la fase 1 .

El sistema de información conformado por información (indicadores) y otros resultados de las investigaciones generadas por las universidades y otras entidades participantes, la información contenida en éste deberá ser comunicada a la sociedad. 


\section{CONCLUSIONES}

Se reconoce a nivel mundial que el sector turístico, manejado sustentablemente, genera riqueza en los espacios en los que esta actividad económica tiene incidencia. Para que el turismo se desarrolle en el Ecuador es necesario generar conocimiento sobre el sector y que necesariamente no está relacionado con el ámbito técnico, sino en aquellos saberes que complementan su comprensión y acceso.

La propuesta de creación e implementación de un observatorio de turismo posibilita la generación de información a través de la investigación aplicada y el acceso a ésta, potenciando la inclusión de sectores sociales, que carecen de un sistema educativo occidentalizado y que nos les permite acceder a sistemas de información alineados. El observatorio pretende ser una herramienta de comunicación que permita recabar experiencias que generen otro tipo de conocimientos que no estén circunscritos a los ámbitos académico y técnico.

Dentro del ámbito educativo el aporte de un observatorio generará información relevante para medir, evaluar y proponer mejoras en la práctica académica universitaria en el sector del turismo.

La construcción de indicadores técnicamente estructurados para medir la productividad y competitividad de los múltiples productos turísticos del país será un aporte interesante para evaluar criterios de inversión pública y privada. Otro aporte importante constituirá también la investigación y evaluación de la relación entre las políticas públicas en materias de leyes, incentivos y obras en el sector turístico con la realidad que viven quienes constituyen el sector turístico del Ecuador.

Para lograr estos objetivos, es importante reconocer que se requiere una gran capacidad de organización del talento humano y materiales involucrados y sobre todo del interés y participación de las autoridades y docentes de la PUCE y de las universidades invitadas. Se prevé una importante actividad en cuanto a desarrollo conceptual, planificación y operación de la investigación así como una gran participación en el trabajo de campo llevado a cabo por los estudiantes y profesores de las universidades participantes.

Finalmente, para que el observatorio cumpla lo planificado es necesario transmitir con exactitud la importancia de los objetivos del observatorio a todos los integrantes del mismo, así como lograr el compromiso para llevar a cabo todas las actividades y la operatividad que se requiera; aspectos fundamentales para el éxito de programas de investigación. 


\section{BIBLIOGRAFIA}

Clacso. (2013). "Portal de observatorios". Recuperado el 25 de noviembre del 2013, de http://www.clacso-posgrados.net/portal_observatorios/

Escotet, Miguel Ángel. (2002). Desafios de la educación superior en el siglo de la incertidumbre. Cuadernos de Investigación en la Educación, No.18. Puerto Rico: Universidad de Puerto Rico.

Desrosières, Alain. (1996). Reflejar o Instituir: la invención de los indicadores estadísticos. Revista de técnicas, métodos e instrumentos de investigación en Ciencias Humanas: No.4

Farné, Stefano. (2011). Observatorios del mercado de trabajo: ¿Qué son y cómo funcionan? Revista Economía Institucional, No. 24.

Fazenda, Nuno. (2013). "Fortalezas y debilidades del turismo en el mundo, una oportunidad para el desarrollo de observatorios turísticos". Observatorio Turístico del Estado de Guanajuato. Recuperado el 25 de noviembre del 2013 en: http://www.observatorioturistico.org/publicaciones/ver_todos/15

Gibbons, M., Limoges, Camille., Nowotny, Helga., Schwartzman, Simon., Scott, Peter., Trow, Martin. (1997). La nueva producción del conocimiento. La dinámica de la ciencia y la investigación en las sociedades contemporáneas. Barcelona: Ediciones Pomares-Corredor, S.A.

Malagón, Ángela (Eds.). (2010). Observatorios de Desarrollo: Conceptos, metodologías y experiencias. Observatorio de Desarrollo Municipal, Asociación de Municipios Región Cibao Norte, Federación Dominicana de Municipios.

Ministerio de Turismo. (2013). "Estadísticas turísticas". Recuperado el 7 de enero del 2014 desde http://servicios.turismo.gob.ec/index.php/?option=com_ content\&view $=$ article\&id

Muñoz y Torrent, Xavier. (2003). "Observatorios socioeconómicos locales y planificación estratégica". Jornadas sobre desarrollo y empleo para entidades de tamaño intermedio. Observatorio Económico y Social de Terrassa. Recuperado en http://www2.terrassa.cat/laciutat/xifres/estudis/observatorios iru

Organización de los Estados Iberoamericanos. (2011). "Observatorio de la educación iberoamericana”. Recuperado el 22 octubre del 2013 en http://www.oei.es/ observatorio/observatorio.htm

Organización Mundial del Turismo. (2013). Panorama OMT del Turismo internacional. $O M T$. Recuperado en http//www.unwto.org/pub

Pérez Rojas, Julia Andrea. (2010). Diseño de un observatorio ambiental para el sector de curtiembres estudio de caso: Curtiembres de Villapinzón y Chocontá. Tesis de Maestría, Universidad Nacional de Colombia. Recuperada de Repositorio digital de la UN (Núm. 8690).

Phélan, M. (2007). La red de observatorios locales de Barcelona, España. Fermentum, 48, (96-122). 
Tünnermann, Carlos y de Souza Chaui, Marilena. (2003). Desafíos de la Universidad en la sociedad del Conocimiento, cinco años después de la Conferencia Mundial sobre Educación Superior. Comité Científico Regional para América Latina y el Caribe del Foro de la UNESCO. Forum. Occasional Paper Series: paper no. 4/5

Tünnermann, Carlos. (2011). Las conferencias regionales y mundiales sobre educación superior dela UNESCO y su impacto en la educación superior en América Latina. Unión de Universidades de América Latina y el Caribe. Universidades: vol. LX. núm. 47, (31-46).

Secretaría Nacional de Planificación y Desarrollo. (2013)."Transformación de la Matriz Productiva". Recuperado el 25 de noviembre en http://www.planificacion.gob. ec/wp-content/uploads/downloads/2013/01/matriz_productiva_WEBtodo.pdf.

World Economic Forum (2013) "Travel and Tourism Competitiveness". Recuperado el 25 de noviembre en http://www.weforum.org/issues/travel-and-tourismcompetitiveness

Recibido: $17 / 01 / 2013$

Aceptado: 20/03/2013

Arbitrado anónimamente. 